\title{
Advance ligation to facilitate pancreaticojejunostomy following pancreaticoduodenectomy by dilating the main pancreatic duct
}

\author{
Masayuki Honda ${ }^{1,2,3}$, Masayoshi Hioki ${ }^{1}$, Hiroshi Sadamori ${ }^{1}$, Kazuteru Monden ${ }^{1}$, Go Wakabayashi ${ }^{3}$, \\ Norihisa Takakura ${ }^{1}$ \\ ${ }^{1}$ Department of Surgery, Fukuyama City Hospital, Zaocho, Fukuyama, Hiroshima, Japan; ${ }^{2}$ Department of Surgery, Sainokuni Higashiomiya Medical \\ Center, Torocho, Kitaku, Saitama, Japan; ${ }^{3}$ Department of Surgery, Ageo Central General Hospital, Kashiwaza, Ageo, Saitama, Japan \\ Contributions: (I) Conception and design: M Honda, M Hioki; (II) Administrative support: M Honda, M Hioki, K Monden, H Sadamori, N Takakura; \\ (III) Provision of study materials or patients: M Honda, M Hioki, K Monden, H Sadamori; (IV) Collection and assembly of data: $M$ Honda, M \\ Hioki, K Monden; (V) Data analysis and interpretation: M Honda, M Hioki, K Monden, H Sadamori; (VI) Manuscript writing: All authors; (VII) \\ Final approval of manuscript: All authors. \\ Correspondence to: Masayuki Honda, MD. Department of Surgery, Sainokuni Higashiomiya Medical Center, 1522, Torocho, Kitaku, Saitama 331- \\ 8577, Japan. Email: masayuki.honda@shmc.jp.
}

Background: Duct-to-mucosa pancreaticojejunostomy (PJ) after pancreaticoduodenectomy (PD) is technically challenging, particularly in cases of soft pancreas with a nondilated main pancreatic duct (MPD). We propose a novel procedure that involves ligating the pancreas in advance to allow for MPD dilation.

Methods: We compared the data of 16 patients who underwent PD followed by PJ with advance ligation (AL) for soft pancreas with a nondilated MPD with that of 17 patients who underwent a conventional procedure (conventional group) without AL at a single institution between January 2015 and April 2017. MPD diameters were assessed using preoperative computed tomography and intraoperative ultrasonography. Pancreatic consistency was judged intraoperatively. The pancreatic parenchyma and MPD were ligated in advance to allow time for MPD dilation. After AL, we divided the pancreatic parenchyma. AL led to MPD dilation and facilitated PJ.

Results: There were significantly fewer complications in the AL group than in the conventional group $(\mathrm{P}=0.005)$. There were no cases of pancreatitis or death in either group. The mean procedural time for occluding the MPD was $43 \mathrm{~min}$ (range, 21-134 min). The median MPD dilation rate after AL was 1.6 (0.93.8).

Conclusions: AL is a simple, safe, and useful procedure for facilitating PJ.

Keywords: Pancreas; ligation; pancreaticoduodenectomy (PD); pancreatic duct; pancreaticojejunostomy (PJ)

Submitted May 15, 2020. Accepted for publication Sep 30, 2020.

doi: 10.21037 /gs-20-501

View this article at: http://dx.doi.org/10.21037/gs-20-501

\section{Introduction}

Duct-to-mucosa pancreaticojejunostomy (PJ) after pancreaticoduodenectomy (PD) remains technically challenging. Furthermore, soft pancreas and a nondilated main pancreatic duct (MPD) are significant risk factors for the development of postoperative pancreatic fistula (POPF) (1). Advance ligation (AL) to allow for dilation of the MPD was gradually introduced in 2015 for cases of soft pancreas with a nondilated duct at our institution. In this study, we report the preliminary surgical outcomes for this novel technique, which facilitates PJ in patients with soft pancreas and a nondilated MPD. We present the following article in accordance with the STROBE reporting checklist (available at http://dx. doi. org/10. 21037/gs-20-501). 
A

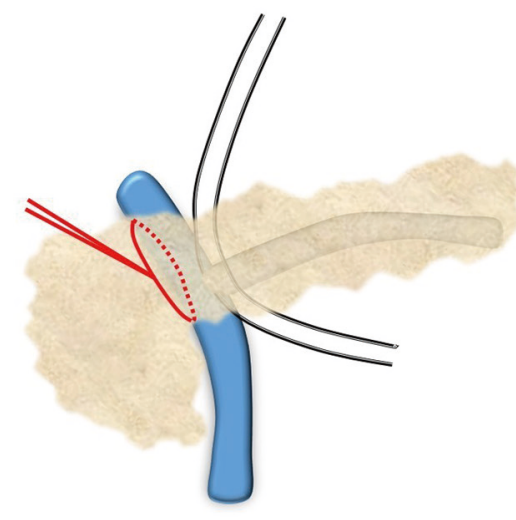

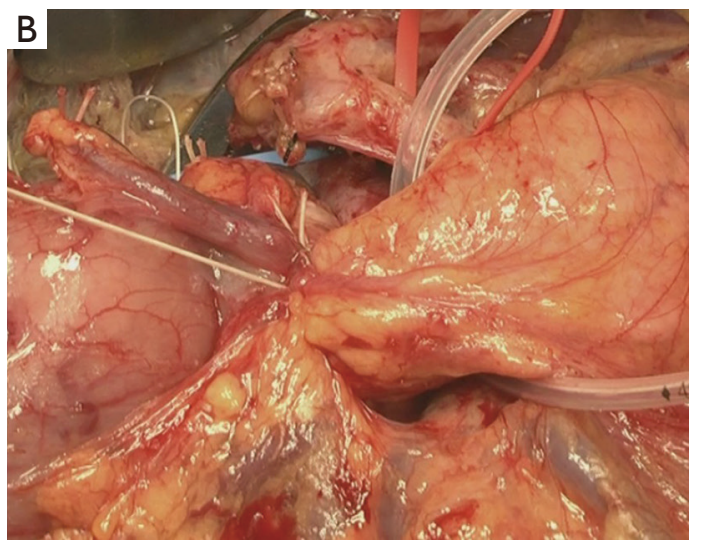

Figure 1 Advance ligation; the pancreatic parenchyma above the right edge of the portal vein was ligated with a 1-0 silk thread in advance (red line). (A) Schema; (B) intraoperative image.
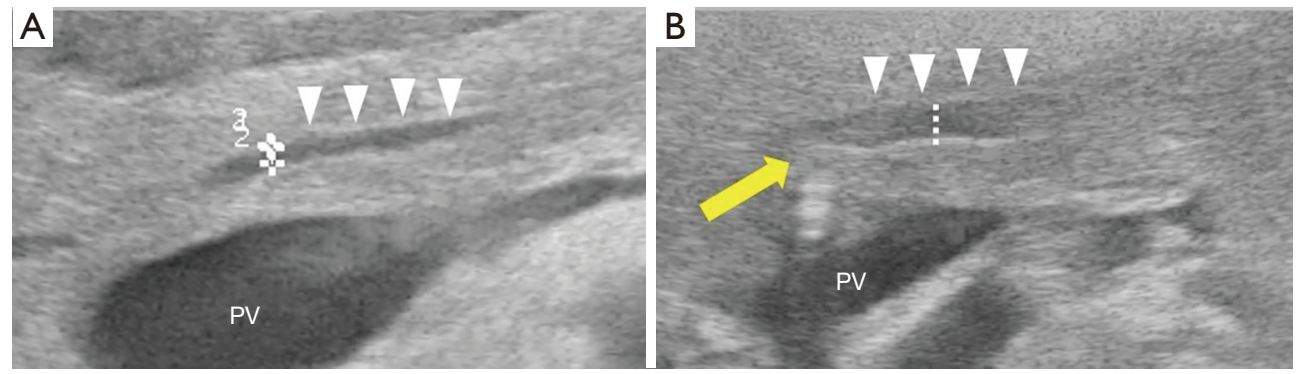

Figure 2 Main pancreatic duct diameters measured with perioperative ultrasonography. (A) Before advance ligation; (B) immediately before dissection of the pancreas. Dashed line, cross: measurement distance of each main pancreatic duct. PV: portal vein, main pancreatic duct (arrowhead), ligation point (arrow).

\section{Methods}

\section{Patients}

Between January 2015 and May 2017, 98 consecutive patients underwent PD followed by PJ for hepatobiliary or pancreatic diseases at the Fukuyama City Hospital. Patients who required hepato-PD or vessel reconstruction were excluded. The pancreatic consistency was intraoperatively judged as soft or hard, and a nondilated duct was defined by an MPD diameter of $<3 \mathrm{~mm}$ on preoperative axial computed tomography (CT). In patients who underwent the conventional procedure, ligation was immediately performed following division of the pancreas. In the AL group, ligation was performed earlier; thus, it was referred to as AL. Clinical data regarding the perioperative course, including postoperative morbidity and mortality, were prospectively collected for all patients. The incidence of POPF was prospectively analyzed according to the
International Study Group on Pancreatic Fistula (ISGPF) criteria (2). This study was conducted in accordance with the Declaration of Helsinki (as revised in 2013). The study was approved by the ethics committee of Fukuyama City Hospital (approval \#320) and individual consent for this retrospective analysis was waived.

\section{AL procedure}

In the $\mathrm{AL}$ group, the pancreatic parenchyma above the right edge of the portal vein was gently ligated using a thick thread (1-0 silk) in advance during PD (Figure 1). Ligation was carefully performed to avoid tearing of the soft pancreatic tissue. A $360^{\circ}$ ligation of the pancreatic parenchyma artificially occluded the MPD. After allowing some time for MPD dilation, we divided the pancreatic parenchyma. The MPD diameter was measured using ultrasonography before pancreatic ligation and immediately 
Table 1 Preoperative and operative data for patients who underwent pancreaticojejunostomy with advance ligation (AL) or conventional ligation

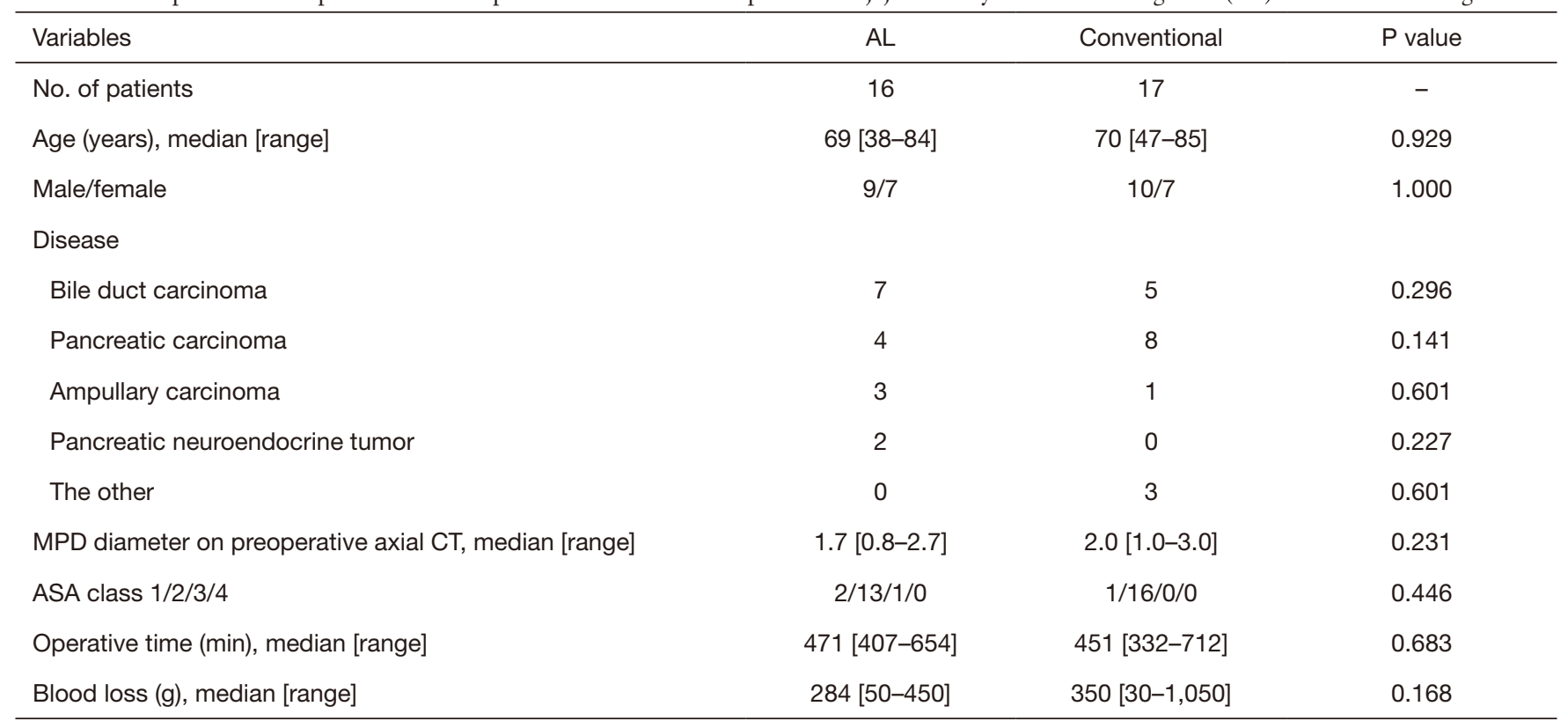

MPD, main pancreatic duct; CT, computed tomography; ASA, American Society of Anesthesiologists.

before dissection (Figure 2). The time between ligation and dissection was recorded. After dissection of the pancreas, a stent was inserted into the MPD through the incision in the pancreatic parenchyma; this prevented not only exposure of the pancreatic juice from the remnant pancreas but also reshrinkage of the MPD.

Following en bloc resection of the pancreatic head, duodenum, bile duct, and gallbladder with the distal portion of the stomach, PJ was performed using the modified Blumgart method (3) with or without transanastomotic pancreatic stenting, depending on the surgeon's preference.

\section{Postoperative management}

An oral diet was resumed on postoperative day (POD) 3. Serum amylase and amylase concentration in fluid collected from the peripancreatic drain were measured on PODs 1, 3 , and 5. The drain was removed on POD 4 in patients who showed no signs of intra-abdominal infection from fluid collected from the peripancreatic drain.

\section{Statistical analyses}

All data were statistically analyzed using SPSS 22.0 statistical software (IBM, Armonk, New York, USA). Comparisons between the two groups were performed using the Chi-squared or Fisher's exact test for categorical data and the Mann-Whitney $U$ test for continuous data. Continuous variables are reported as median (range). A P value of $<0.05$ was considered statistically significant.

\section{Results}

Of the 98 screened patients, 33 (20 men, 13 women) were included in this study. AL during PD was successfully performed in 16 patients, while the conventional procedure was performed in the remaining 17 (Table 1). The two groups showed no differences in patient age, male-to-female ratio, operative indications, MPD diameter on preoperative axial CT, American Society of Anesthesiologists physical status, total operative time, and total intraoperative blood loss. A PJ stent was inserted in 15 (94\%) patients in the AL group and 14 (82\%) in the conventional group, with no significant difference between the two groups. The AL group showed a higher rate of Clavien-Dindo grade I-II postoperative complications $(\mathrm{P}=0.037)$, but a significantly lower rate of grade III-IV complications $(\mathrm{P}=0.005$; Table 2). Pancreatic fistula was noted in $0 \%$ of patients in the AL group and $29 \%$ of those in the conventional group $(\mathrm{P}=0.026)$. There was no significant between-group difference in the median serum amylase levels on PODs 1 and 3. Intra-abdominal hemorrhage occurred in one patient 
Table 2 Postoperative complications in patients who underwent pancreaticojejunostomy with advance ligation (AL) or conventional ligation



${ }^{\dagger}$, less than 1 month after discharge. POPF, postoperative pancreatic fistula; ISGPS, the 2016 International Study Group for Pancreatic Surgery; POD, post-operative day.

in the conventional group on POD 2, and was successfully managed by reoperation. The length of hospital stay and readmission rate were similar in the two groups. One patient in the AL group was readmitted to the hospital within 1 month because of delayed gastric emptying. There were no operative mortalities in either group.

In the AL group, the median MPD diameter before $\mathrm{AL}$ was $2.0 \mathrm{~mm}$ (range, $1.0-3.5 \mathrm{~mm}$ ). The MPD orifice was occluded through AL in a mean of 43 min (range, 21$134 \mathrm{~min}$ ). The median MPD diameter before the dissection of the pancreas was $2.8 \mathrm{~mm}$ (range, 1.4-4.1 $\mathrm{mm}$ ), and the median MPD dilation rate after AL was 1.6 (range, 0.9-3.8).

\section{Discussion}

This report describes the preliminary results of AL in PD for soft pancreas with a nondilated MPD, which enables MPD dilation without severe morbidity related to failure of the PJ. This approach could be applicable to various PJ methods during PD, including the Kakita (4), modified Blumgart (3), and two-layer techniques (5), without requiring specialized instruments, regardless of laparotomy 
or laparoscopy.

The MPD dilation may, theoretically and intuitively, render suturing of the PJ simpler. The soft texture of the pancreas, which leads to easy deformation rather than a higher risk for POPF after PD, allowed the MPD to be easily occluded by AL, resulting in a median 1.6x dilation. No patients in the present study developed grade $\mathrm{B}$ or $\mathrm{C}$ POPF despite having both soft pancreas and a nondilated MPD. The dilated MPD, which made the PJ anastomosis easier, may be one of the factors contributing to POPF reduction.

Because AL induced an artificial obstruction of the MPD, we were concerned about postoperative pancreatitis occurring in the remnant pancreas. The duration of $\mathrm{AL}$ (median $43 \mathrm{~min}$ ) led to high levels of median serum amylase on POD 1 (407 IU/L), but the level returned to normal in all patients on POD 3 (58 IU/L). This time was sufficient to expand the MPD, but not likely to cause pancreatitis.

This study has several limitations associated with the single-institution retrospective design and the small sample size, which prevents conclusions regarding the safety of the method. To overcome these limitations, a further prospective large-scale study is needed to compare the outcomes between the patients who underwent PD with and without AL. Nonetheless, this surgical technique may facilitate PJ.

\section{Conclusions}

To conclude, we introduced a new procedure, AL, during PD to facilitate PJ by dilating the MPD intraoperatively. AL was simple, safe, and effective for performing anastomoses in the soft pancreas with a nondilated MPD. While definitive conclusions will require a randomized study with a control cohort, the present report may demonstrate the usefulness and feasibility of AL with no severe complications.

\section{Acknowledgments}

We would like to thank Editage (www.editage.com) for English language editing.

Funding: None.

\section{Footnote}

Reporting Checklist: The authors have completed the STROBE reporting checklist. Available at http://dx. doi. org/10. 21037/gs-20-501

Data Sharing Statement: Available at http://dx. doi. org/10. 21037/gs-20-501

Peer Review File: Available at http://dx. doi. org/10.21037/ gs $-20-501$

Conflicts of Interest: All authors have completed the ICMJE uniform disclosure form (available at http://dx. doi. org/10. 21037/gs-20-501). The authors have no conflicts of interest to declare.

Ethical Statement: The authors are accountable for all aspects of the work in ensuring that questions related to the accuracy or integrity of any part of the work are appropriately investigated and resolved. This study was conducted in accordance with the Declaration of Helsinki (as revised in 2013). The study was approved by the ethics committee of Fukuyama City Hospital (approval \#320) and individual consent for this retrospective analysis was waived.

Open Access Statement: This is an Open Access article distributed in accordance with the Creative Commons Attribution-NonCommercial-NoDerivs 4.0 International License (CC BY-NC-ND 4.0), which permits the noncommercial replication and distribution of the article with the strict proviso that no changes or edits are made and the original work is properly cited (including links to both the formal publication through the relevant DOI and the license). See: https://creativecommons.org/licenses/by-nc-nd/4.0/.

\section{References}

1. Tani M, Kawai M, Hirono S, et al. A prospective randomized controlled trial of internal versus external drainage with pancreaticojejunostomy for pancreaticoduodenectomy. Am J Surg 2010;199:759-64.

2. Bassi C, Marchegiani G, Dervenis C, et al. The 2016 update of the International Study Group (ISGPS) definition and grading of postoperative pancreatic fistula: 11 Years After. Surgery 2017;161:584-91.

3. Hirono S, Kawai M, Okada KI, et al. Modified Blumgart Mattress Suture Versus Conventional Interrupted Suture in Pancreaticojejunostomy During Pancreaticoduodenectomy: Randomized Controlled Trial. Ann Surg 2019;269:243-51.

4. Kakita A, Takahashi T, Yoshida M, et al. A simpler and 
more reliable technique of pancreatojejunal anastomosis. Surg Today 1996;26:532-5.

5. Pratt WB, Maithel SK, Vanounou T, et al. Clinical and economic validation of the International Study Group of Pancreatic Fistula (ISGPF) classification scheme. Ann Surg 2007;245:443-51.

Cite this article as: Honda $M$, Hioki $M$, Sadamori H, Monden K, Wakabayashi G, Takakura N. Advance ligation to facilitate pancreaticojejunostomy following pancreaticoduodenectomy by dilating the main pancreatic duct. Gland Surg 2021;10(1):59-64. doi: 10.21037/gs-20-501 